\title{
Mass production and application of activation tagged hairy root lines for functional genomic of secondary metabolism in ginseng
}

\author{
Dong-Woog Choi - Hwa-Jee Chung - Suk Min Ko - Dong Soo In · JiSook Song • \\ Sung-Sick Woo $\cdot$ Jang R. Liu
}

Received: 21 September 2009 / Accepted: 25 September 2009

(c) Korean Society for Plant Biotechnology

\begin{abstract}
Activation tagging that uses T-DNA vectors containing multimerized transcriptional enhancers from the cauliflower mosaic virus (CaMV) 35S gene is a powerful tool to determine gene function in plants. This approach has been successfully applied in screening various types of mutations and cloning the corresponding genes. We generated an activation tagged hairy root pool of ginseng (Panax ginseng C.A. Meyer) in an attempt to isolate genes involved in the biosynthetic pathway of ginsenoside (triterpene saponin), which is known as the major active ingredient of the root. Quantitative and qualitative variation of ginsenoside in activation tagged hairy root lines were profiled using LC/MS. Metabolic profiling data enabled selection of a specific hairy root line which accumulated ginsenoside at a higher level than other lines. The relative expression level of several genes of triterpene biosynthetic pathway in the selected hairy root line was determined by real time RT-PCR. Overall results suggest that the activation tagged ginseng hairy root system described in this study would be useful in isolating genes involved in a complex metabolic pathway from genetically intractable plant species by metabolic profiling.
\end{abstract}

D.-W. Choi

(Department of Biology Education, Chonnam National University, Gwangju, 500-757, Korea)

H.-J. Chung $\cdot$ S. M. Ko $\cdot$ D. S. In

(Eugentech Inc. 52-Oun Dong, Yuseong, Daejeon, 305-333, Korea)

J. S. Song $\cdot$ S.-S. Woo

(UniGen Inc., San 80, Songjeong-ri, Byeongcheon-myeon,

Cheonan, Chungcheongnam-do, 330-863, Korea)

J. R. Liu ( $\bowtie)$

(Korea Research Institute of Bioscience and Biotechnology

(KRIBB), Daejeon, 305-333, Korea)

e-mail: jrliu@kribb.re.kr

\section{Introduction}

The powerful tool to dissect a genetic pathway and to determine gene function is generation and analysis of the mutant lines disrupted interesting pathway or phenotype. T-DNA insertion mutagenesis has provided a more rapid way to find a mutated phenotype and gene (Azpiroz-Leehan and Feldmann 1997; Bouchez and Hofte 1998; Bouche and Bouchez 2001). However, the mutation by insertional mutagenesis usually causes loss of function, and results in recessive mutation, which makes it difficult to screen a desire mutant phenotype in primary transformants. Insertion mutagenesis approach is not applicable to plant species in which a high efficiency transformation procedure has not been established and genes that act redundantly (Bouche and Bouchez 2001).

Activation tagging, an alternative approach to generate gainof-function mutation, can circumvent many disadvantages of loss-of-function approaches (Weigel et al. 2000; Walden et al. 1994). In activation tagging system, T-DNA that carries enhancer element of CaMV 35S promoter is used as a mutagen and introduced into plant cell. Upon random integration of enhancer element into the genome, flanking plant sequences can be transcriptionally activated, resulting in a gain-of-function mutation. Mutations created by activation tagging are usually dominant or semi-dominant. Therefore, in contrast to loss-of- function mutation, mutants generated by activation tagging allow direct selection for desired phenotypes in primary transformants. Activation tagging has been successfully applied in screening various types of mutations and cloning the corresponding genes (Weigel et al. 2000; Kakimoto 1996; Borevitz et al. 2000; Huang et al. 2001), but limited in several plant species in which a high efficiency genetic transformation system is available.

Plants produce a high diversity of secondary metabolites that 
play important biological roles in adaptation to environments. Plant secondary metabolites have been used as dyes, flavors, drugs, and other useful agents (Croteu et al. 2000). However, knowledge about biosynthetic pathway of plant secondary metabolism is still limited (Dixon 1999; Pichersky and Gang 2000). A conventional mutation approach is not easily applicable in elucidating genes for plant secondary metabolism. It is because secondary metabolites are not usually accumulated at high levels in plants so that a deletion mutant of a gene encoding an enzyme for catalyzing biosynthesis of a secondary metabolite would exhibit no detectable phenotype (Croteu et al. 2000; Pichersky and Gang 2000). The challenge now is to develop an excremental system for functional genomics of secondary metabolism in those plant species that are rich source of natural products. The application of activation tagging system in genetically intractable plant species to isolate gene involved in secondary metabolism was reported by van der Fits et al. (2001). They screened activation tagged calluses of Catharathus roseus and isolated a gene encoding ORCA3, AP2 domain transcriptional regulator for several gene involved in biosynthesis of terpene indole alkaloid (van der Fits and Memelink 2000).

The root of ginseng (Panax ginseng C.A. Myer) is widely used as a traditional medicine since ancient time owing to stimulating and tonic properties. Ginsenosides, triterpene saponins are known as the major active compounds of the root. To dissect and isolate genes involved in ginsenoside biosynthesis, we generated activation tagged ginseng hairy root lines and examined the ginsenoside profile of each line using liquid chromatography/mass spectrometry (LC/MS) system. Here we report an activation tagged ginseng hairy root system for functional genomics of secondary metabolism.

\section{Materials and Methods}

\section{Construction of activation tagging vector $\mathrm{pKH} 01$}

A fragment including the tetramer enhancer of the cauliflower mosaic virus (CaMV) $35 \mathrm{~S}$ promoter from pSKI015 was PCR amplified and subcloned into cloning vector pCR2.1 TOPO (Invitrogen, USA). To PCR amplification of enhancer fragment, following oligonucleotide primers were used: 5 '-TC TAGAACTAGTGGATCCCCAACATG-3', 5'-CTGCAG AATATATCCTGTCAAACACTG-3'. Enhancer fragment on pCR2.1 TOPO vector was digested with BamHI/PstI and subcloned into BamHI/PstI site of pCAMBIA2300, resulted in pKH01 (Fig. 1, A). pKH01 was introduced into Agrobacterium rhizogenes R-1000 obtained from KCTC (Korean Collection for Type Culture) of the Korea Research Institute of Biotechnology and Bioscience (Daejeon, Korea).

Induction and generation of activation tagging line

Stratified seeds of ginseng (Panax ginseng C.A Meyer, cv. KG101 [Chunpoong]) were used as starting plant material. After removed seed coat, embryos were sterilized with $70 \%$ ethanol for $30 \mathrm{~min}$ and then $0.4 \%$ sodium hypochlorite for 15 min before rinsing sterilized distilled water. Disinfested seeds were placed on 1/2 Murashige and Skoog (MS) (1962) medium and maintained at $25^{\circ} \mathrm{C}$. After 3 weeks of incubation, cotyledons and hypercotyls were excised from germinated seedlings and sliced into less than $1 \mathrm{~cm}^{3}$ explants for cocultivation with A. rhizogenes strain R-1000 harboring the activation tagging vector $\mathrm{pKH} 01$. Explants were dipped into Agrobacterium suspension for 30 minute and transferred on solidified $1 / 2 \mathrm{MS}$ medium with $0.4 \%$ Gelite, and then maintained at $27^{\circ} \mathrm{C}$ in dark. After $48 \mathrm{hrs}$ of incubation, cocultivated explants were washed with $1 / 2$ MS liquid medium with $800 \mathrm{mg} / \mathrm{L}$ cefotaxime and transferred into selection medium comprising $1 / 2 \mathrm{MS}$ medium with $400 \mathrm{mg} / \mathrm{L}$ of cefotaxime and $100 \mathrm{mg} / \mathrm{L}$ of kanamycin. Selection medium was replaced every 3 weeks to eliminate excessive Agrobacterium cells. After cocultivation of 4-8 weeks, hairy roots were generated at the infection site.

To establish independent hairy root lines, hairy roots were cut from each of explants cocultivated with Agrobacterium. When more than 2 hairy root lines were excised from a single

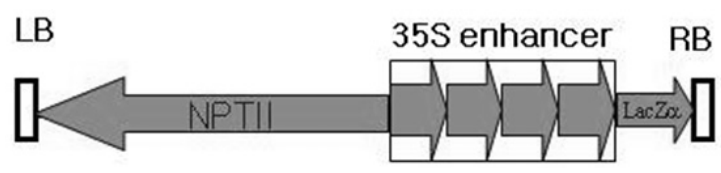

(A)

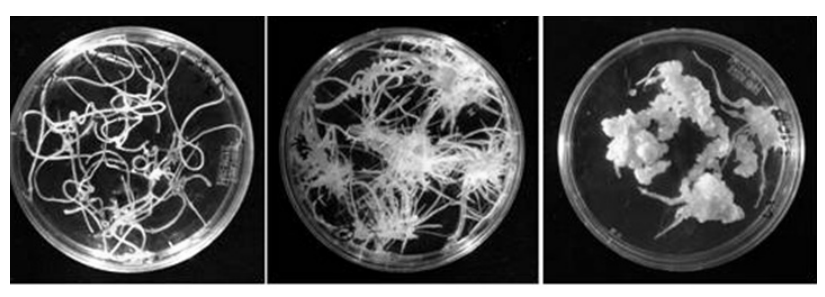

(B)

Fig. 1 A. Map of activation tagging vector pKH01. Tetramer of CaMV 35S enhancer element from pSKI015 was subcloned into pCAMBIA2300. B. Three typical phenotype of ginseng hairy root. Type 1 was a low branching hairy root type, which had a slow growth pattern and brownish color except a newly developing part. The second group had a typical hairy root phenotype with highly branching and rapid growth. The third group had a callus-like phenotype with few branching 
explant, hairy roots developed from different infection sites on the explant were selected so that different T-DNA tagging lines were isolated. Hairy root lines were maintained on 1/2 Schenk and Hildebrandt (1972) medium without growth regulators at $27 \mathrm{C}$ in the dark and subcultured at every 4 week interval. Only hairy roots transformed with A. rhizogenes were able to grow on culture medium without growh regulators, which is a typical hairy root phenotype. Ginseng roots never appeared on control disks on which were not infected with A. rhizogenes.

Extraction and analysis of ginsenosides

Twenty milligram of freeze-dried hairy roots was subjected to extraction with $80 \% \mathrm{MeOH}$. Authentic ginsenosides Rb1, $\mathrm{Rb} 2, \mathrm{Rc}, \mathrm{Rd}, \mathrm{Re}, \mathrm{Rf}$, and Rg1 purchased from INDOFINE Chemical Company, Inc. (Somerville, NJ, USA) were used to identify ginsenosides from root extracts by comparison. Root extracts were analyzed using HPLC-SSI-MS system (Hitachi's 3DQ LC/Ion Trap system, Hitachi High Technologies America, Inc., San Jose, USA) with sonic spray ionization interface. HPLC column was connected to UV spectrometer followed by the sonic spray interface including of capillary sheath and evaporating plates in the mass spectrometer. The cover plate was heated to $250^{\circ} \mathrm{C}$ and sampling orifice was heated to $170^{\circ} \mathrm{C}$. The spray voltage was set to $1 \mathrm{kV}$ and the drift voltage between sample orifice and was $70 \mathrm{~V}$. The nitrogen, carrier gas gauge pressure was 4.5 bar, and the helium, collision gas pressure was 2.6 bar. Mass spectrometer conditions were optimized in order to achieve maximum sensitive utilizing of the buffer solution with $1 \%$ TEA from end of column to mass in flow line. The calibration of the mass range $(200 \sim 1200 \mathrm{Da})$ was performed in positive ion mode, using the standard calibration procedure and compounds (TBA, Reserpine, Bredkinin). Full scan mass spectra were acquired from 300-1200 amu at unit mass resolution. For stepwise fragmentation experiments, datadependent scanning was chosen with the wideband activation turned off. The normalized collision energy was set to $50 \%$.

Expression analysis of triterpene biosynthetic genes by real time RT-PCR

Total RNA was extracted from three weeks old hairy root after subculture using Trizol reagent and treated with RNase-free DNase (Life Technology, USA) at room temperature for $15 \mathrm{~min}$. DNase was inactivated by adding EDTA ( $2.5 \mathrm{mM}$ final concentration) and heating to $65^{\circ} \mathrm{C}$ for $10 \mathrm{~min}$. A first strand cDNA was synthesized from $5 \mu \mathrm{g}$ of DNase treated total RNA with oligo $d(T)$ primer using
SuperScript II (Life Technology, USA) according to manufactur's manual. The reaction mixture contains $50 \mathrm{mM}$ Tris- $\mathrm{HCl}$ (pH 8.3), $75 \mathrm{mM} \mathrm{KCl}, 3 \mathrm{mM} \mathrm{MgCl}_{2}, 10 \mathrm{mM} \mathrm{DTT}$, $0.5 \mathrm{mM}$ each dNTP. The reaction mixture was incubated at $65^{\circ} \mathrm{C}$ for $5 \mathrm{~min}$, and then 1 unit of RNase inhibitor and 200 unit of SuperScript II were added before incubation at $42^{\circ} \mathrm{C}$ for $1 \mathrm{hr}$. The enzyme was inactivated by heating at $95^{\circ} \mathrm{C}$ for $5 \mathrm{~min}$. The fist strand cDNA reaction mixture was 10 times diluted with $\mathrm{dH}_{2} \mathrm{O}$ and sore at $-20^{\circ} \mathrm{C}$. For the RT-PCR reaction, $1 \mu \mathrm{l}$ of diluted the first strand cDNA reaction mixture was used.

Quantitative PCR was performed in DNA Engine Opticon (MJ Reasherch, USA) using QuantiTect SYBR Green PCR Kit (Quiagen, German). Relative expression level of five genes involved in triterpene biosynthesis, fernasylphyrophosphate synthase (FPS), squalene synthase (SQS), squalene epoxidase (SE), $\beta$-amyrin synthase ( $\beta A S$ ), and cycloartenol synthase $(\mathrm{CS})$ were analyzed. Actin gene was used as internal standard control. The target gene specific primers used for PCR are following. 5'-CAATTCGGAATGAACCCTT-3' and 5'-TTGCCTTTGTGATGTCTGG-3' for FPS, 5'-AAA GCTCTTCCATGCCTCTGGG-3' and 5'-CTGCCTTGGC TGAGTTTTCCTC-3' for SQS 5'-TGGTTGATGTGCCT GGAC-3' and 5'-CACCCGGAGTTGGATGAG-5' for SE, 5'-TGCCAAGGAGGACATCTA-3' and 5'-TCAACCCA ACAAACAAGC-3' for bAS, 5' - -3' and 5'- -3' for CS. The reaction mixture contains $1 \mathrm{X}$ Quantitect SYBR green PCR mix, $0.3 \mu \mathrm{M}$ forward and reverse gene specific primer and approximately $10 \mathrm{ng}$ of first strand cDNA. PCR reactions were initiated at $95^{\circ} \mathrm{C}$ for $15 \mathrm{~min}$, followed by 44 cycles of $94^{\circ} \mathrm{C}$ for $30 \mathrm{sec}, 58^{\circ} \mathrm{C}$ for $30 \mathrm{sec}, 72^{\circ} \mathrm{C}$ for $1 \mathrm{~min}$, and then check melting curve of $\mathrm{PCR}$ product by $95^{\circ} \mathrm{C}$ for $3 \mathrm{~min}, 30^{\circ} \mathrm{C}$ for $5 \mathrm{~min}$ and then $65^{\circ} \mathrm{C}$ to $95^{\circ} \mathrm{C}$ at $0.2^{\circ} \mathrm{C}$ increment per 10 second hold. Florescence data collection was done during the $72^{\circ} \mathrm{C}$ cycle step. To account for differences in total RNA present in each sample, the cDNA amount calculated was normalized using the transcript level of an actin gene detected in the same sample.

\section{Results and Discussion}

Generation of ginseng activation tagging lines

We generated 1,100 activation tagged ginseng hairy root lines for functional genomics of ginsenoside metabolism. Hairy roots exhibited different morphological phenotypes. They were classified into three different groups based on root morphology (Fig. 1, B). The first group had low branching phenotype with a low growth rate and moderate branching 
in brownish color except newly developing parts of the root. The second group had typical hairy root phenotype with a high growth rate and high branching. The third group had callus-like phenotype with a low branching. These hairy root phenotypes were maintained during prolonged subculture period. Mallol et al. (2001) also reported different phenotypes in ginseng hairy root. Phenotypic variation in activation tagging hairy root lines in this study does not seem to be ascribed to transcriptional activation by enhance element. Transcriptional activation of gene related in secondary metabolism by enhance element should be analyzed by other trait like metabolic change. Spena et al. (1987) reported that hairy root phenotypes are determined by relative expression level of rol genes, which effect on the metabolism of plant hormones. However, Nilsoon and Olsson (1997) suggest other hypothesis that rol genes might be change hormone sensitivity or signal transduction rather than hormone metabolism in cell. Recent study show that aux gene on TR-DNA also may effect on phenotype of ginseng hairy root, especially to lateral root branching development (Moyano et al. 1999; Mallol et al. 2001). These hypotheses would be worthwhile to study detail for the function of rol and aux genes in the hairy root development.

The application of activation tagging system in genetically intractable plant species was tried using calli (van der Fits and Memelink 2000). Here, we demonstrate of activation tagging can be applied to hairy roots for functional genomics of secondary metabolism. Hairy root have following advantages as material: 1) Hairy root produce high level of secondary metabolites, which detected in roots of wild type plants (Ahn et al. 1996; Bulgakov et al. 1998). 2) Plant regeneration system does not necessary. Generated hairy root lines are subsequentially maintained as primary transformants without regeneration step and used for further analysis. So it is possible to apply to many of other plant species, which have low regeneration system. 3) Hairy root is a differentiated organ and genetically much stable than suspension culture cell or callus.

In this study, activation tagged hairy roots were generated using A. rhizogenes strain R-1000 as host. Hairy roots were selected on kanamycin free medium, since kanamycin inhibits severely ginseng root growth. Therefore, some of activation tagging hairy root line could not contains enhancer element, because A. rhizogenes can transfer either or both of two T-DNA fragments on activation tagging vector $\mathrm{pKH} 01$ and own Ri-Plasmid. We randomly selected 44 hairy root lines and verified rolB gene and $35 \mathrm{~S}$ enhancer fragment by PCR procedure. Among the 44 hairy root lines, 37 lines (84.1\%) contained both of genes, and 7 lines did not contain $35 \mathrm{~S}$ enhancer fragment but had only rolB gene (data not

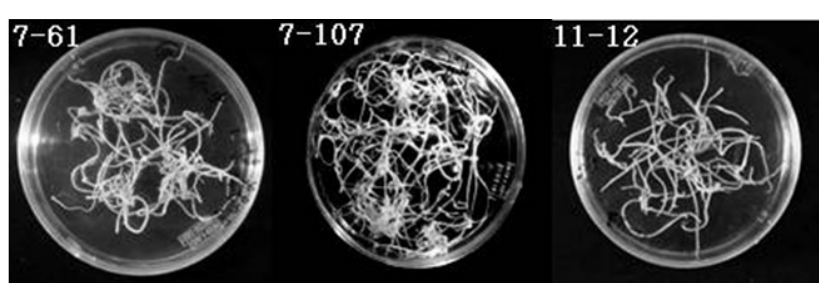

(A)

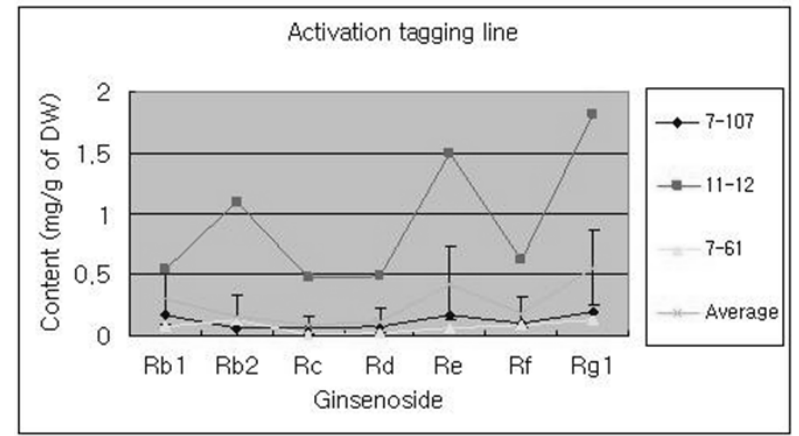

(B)

Fig. 2 Ginsenoside content in activation tagging hairy root lines. A. Seven ginsenoside, four protopanaxadiol ginseng saponins ( $\mathrm{Rb} 1$, $\mathrm{Rb} 2$, Rc,and $\mathrm{Rd}$ ) and three protopanaxatriol ginseng saponins (Re, $\mathrm{Rf}$ and $\mathrm{Rg} 1$ ) in selected activation tagged lines were profiled using LS/MS. Refer to Table 1. Average value was calculated from 60 activation tagging lines. Bars indicate the standard deviation of 60 activation tagging lines. B. Phenotype of selected hairy root lines

shown). It seems that the use of efficient selection marker should be applied to select only activation tagged hairy root line. In addition, it would be required that a new activation tagging vector should be designed with enhancer elements and a rol gene to minimize genetic variation between hairy root lines.

Metabolic profiling of ginsenoside in ginseng hairy root lines

We determined quantitative and qualitative variation of ginsenosides in activation tagged ginseng hairy root lines using LC/MS (Fig. 2). Total 41 putative ginsenoside compounds were detected by LC/MS from ginseng hairy roots (data not shown). In this work, we analyzed only seven ginsenosides comprising four protopanaxadiol ginseng saponins ( $\mathrm{Rb} 1, \mathrm{Rb} 2, \mathrm{Rc}$, and $\mathrm{Rd}$ ) and three protopanaxatriol ginseng saponins (Re, Rf, and Rg1), which were used as standards. Ginsenosides Re and Rg1 were detected at the highest level in most of hairy root lines, which was followed by ginsenoside Rb1. Ginsenosides Rg1 and Rc were detected at the lowest level.

Metabolic profiling data from activation tagging hairy root lines showed that ginsenoside accumulation level was variable 


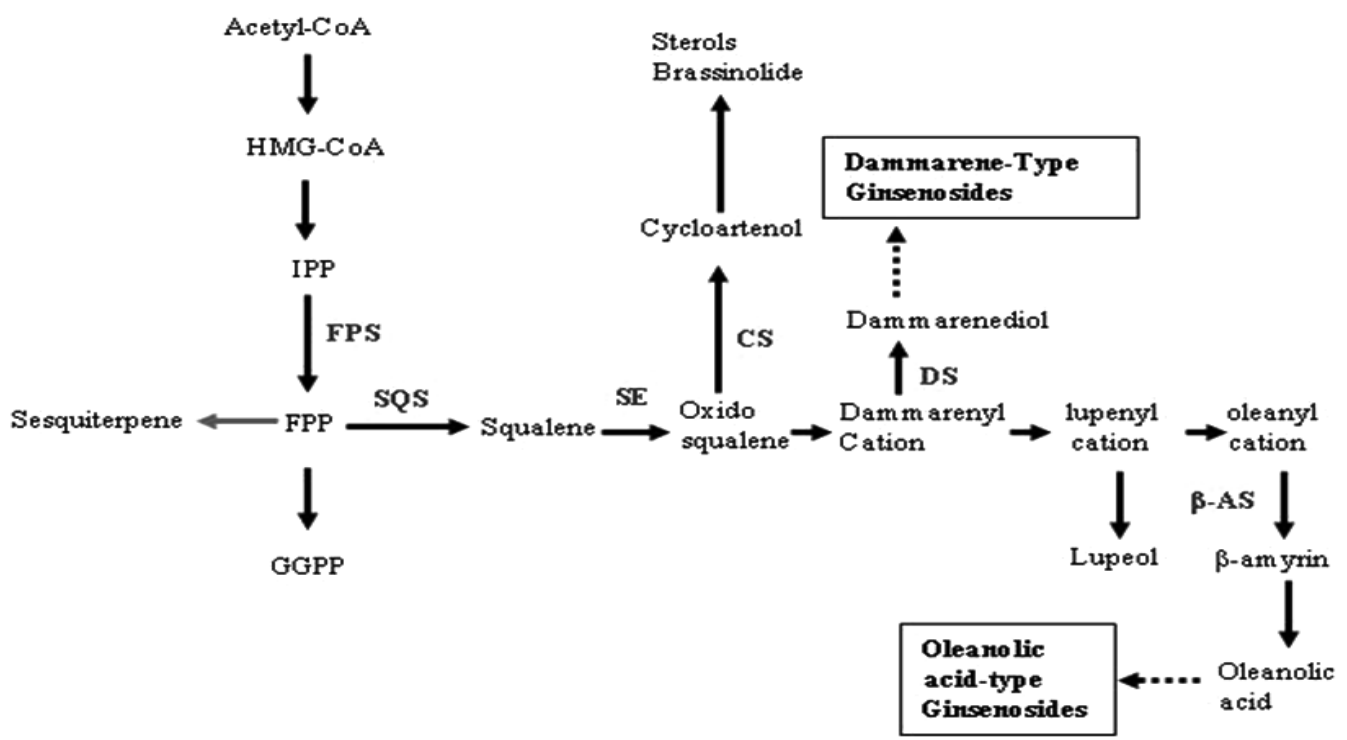

Fig. 3 Ginsenoside biosynthetic pathway. There are two types of ginsenosides, which are biosynthesized by isoprenoid pathway. Farnesyl diphosphate synthase (FPS) is the central branch point of the isoprenoid biosynthesis pathway. Squalene synthase (SQS) and squalene epoxidase (SE) are the first two steps for triterpene biosynthesis. $\beta$-AS is involved in nonsterol triterpene biosynthesis. Cycloartenol synthase (CS) is the first branching point for sterol biosynthesis. DS, dammarenediol synthase; $\beta$-AS, $\beta$-amyrin synthase

among hairy root lines (Fig. 2 B). This result supports previous reports that showed variation of secondary metabolite contents among hairy root lines of ginseng (Mallol et al. 2001) and Atropa belladonna (Bonhomme et al. 2000). However, in this study, the ratio of ginsenosides essentially remained constant among hairy root lines. The average ratio of panaxadiol to panaxatriol glycosdie in R-1000 line was 0.59 . Ratio of panaxadiol glycoside to panaxatriol glycoside is constant in ginsenoside composition in ginseng tissue (Bulgakov et al. 1998). From metabolic profiling data of activation tagging lines, we selected a hairy root line 11-12 that contained the highest content level of ginsenosides. The content of ginsenosides in line 11-12 was 5 times higher than in line 7-61 and 7-107, which had a phenotype similar to line 11-12 (Table 1, Fig. 2B). Relative accumulation of each gensenoside was also different. The level of ginsenoside $\mathrm{Rb} 2$ was significantly higher than other lines. Therefore, it was demonstrated that metabolic profiling of target metabolites using LC/MS would be a method to screen activation-tagging lines for the functional analysis of genes involved in secondary metabolism like the ginsenoside biosynthetic pathway that cannot be easily scored by visual inspection. Metabolites represent the end product of gene expression and metabolic profiling can give a broad view of the biochemical status or biochemical phenotype of an organism that can be directly linked to gene function (Rossener et al. 2001; Raamsdonk et al. 2001). Therefore, metabolic profiling is an emerging powerful tool for functional genomics (Fiehn et al. 2000; Trethewey 2001; Sumner et al. 2002). The use of inhibitors of biosynthetic enzyme may another screening strategy for regulators of metabolism. Recently, Wentzinger et al. (2002) used squalestatin-1 and terbinafine, specific inhibitor for squalene synthase and squalene epoxidase to study regulatory metabolism of sterol branching. Increase of ginsenoside may be accompanied by stimulation of constitutive enzyme activities or activation of genes involved in the ginsenoside biosynthetic pathway.

Expression analysis of triterpene biosynthetic genes in activation tagging hairy root line

We used real-time RT-PCR to examine whether the genes involved in the triterpene biosynthesis pathway are transcriptionally activated in the activation tagged hairy root line 11-12. Previous works reported that there are more than 30 different ginsenoside, triterpene saponins based on two triterpenoids backbone, dammarene and oleanolic acid (Fig. 3). However, the ginsenoside biosynthetic pathway and its regulation are little known. RT-PCR analysis was conducted to determine the transcription level of the three genes involved in biosynthesis of oxidoseuqlene, a common substrate of triterpnoid, farnesyldiphosphate synthase (FPS), squalene synthesis (SS) and squalene epoxidase (SE), and two different oxidosqualene cyclases, cycloartenol synthase (CS) and $\beta$ -amyrin synthase ( $\beta$-AS) (Fig. 3).

Expression levels of target genes in line 11-12 were compared with those in two hairy root lines, 7-61 and 7-107, which had a similar phenotype but a lower level of ginsenoside accu- 
Table 1 Ginsenosides in activation tagging ginseng hairy root lines

\begin{tabular}{ccccccccc}
\hline \multirow{2}{*}{ Line } & \multicolumn{7}{c}{ Ginsenoside $(\mathrm{mg} / \mathrm{g}$ of dry weight) } & \multirow{2}{*}{$\mathrm{PD} / \mathrm{PT}^{2)}$} \\
\cline { 2 - 7 } & $\mathrm{Rb} 1$ & $\mathrm{Rb} 2$ & $\mathrm{Rc}$ & $\mathrm{Rd}$ & $\mathrm{Re}$ & $\mathrm{Rf}$ & $\mathrm{Rg} 1$ & 0.914 \\
$7-61$ & 0.081 & 0.128 & 0.020 & 0.028 & 0.062 & 0.092 & 0.128 & 0.809 \\
$7-107$ & 0.173 & 0.067 & 0.064 & 0.082 & 0.169 & 0.109 & 0.200 & 0.664 \\
$11-12$ & 0.545 & 1.093 & 0.482 & 0.494 & 0.501 & 0.625 & 1.811 & 0.596 \\
Average $^{1)}$ & 0.299 & 0.165 & 0.089 & 0.125 & 0.428 & 0.191 & 0.559 & \\
\hline
\end{tabular}

1) average from 60 AT lines

2) $(\mathrm{Rd}+\mathrm{Rc}+\mathrm{Rb} 1+\mathrm{Rb} 2) /(\mathrm{Rg} 1+\mathrm{Rf}+\mathrm{Re})$

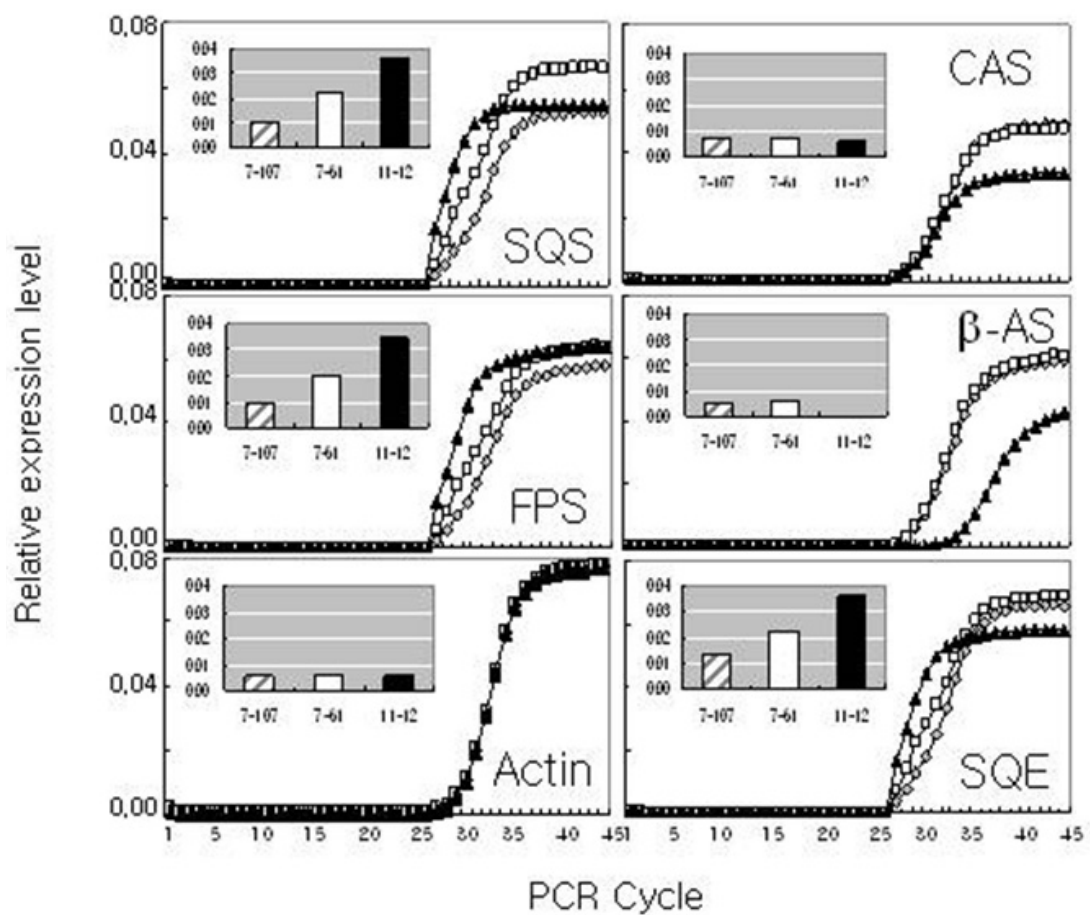

Fig. 4 Expression level of genes in the triterpene biosynthetic pathway in activation tagging hairy root lines. Total RNA isolated from 4 weeks old hairy root lines were used for real time RT-PCR. Five target genes, FPS, SQS, SE, b-AS, and CS are involved in triterpene biosynthesis (see Fig. 3). To account for differences in total RNA present in each sample, the cDNA amount calculated was normalized using the amount of actin gene detected in the same sample. Inset graph shows the relative expression level of target genes at 30 cycles

mulation (Table 1). Expression level of $\beta A S$ gene in line 11-12 was significantly lower than other lines, although $\beta$ amyrin is the precursor of oleanolic acid used for oleanolic type ginsenoside. The $C S$ gene was expressed at a constant level in three hairy root lines. CS is clearly distinct from $\beta$ AS, and produces cycloartenol for first step of sterol biosynthesis, of which biosynthesis starts from triterpenoid squalene. The real time PCR results showed that the expression level of FPS, SQS and SE in hairy root line 11-12 was higher than in hairy root line 7-61 and 7-107 (Fig. 4). These results were coincident with ginsenoside accumulation in these hairy root lines. In general, plant FPS and SS are encoded by a small multigene family and ubiquitously expressed in all of plant tissues (Cunillera et al. 1996; Gaffe et al. 2000; Deva- renne et al. 2002). Increase of accumulation of transcripts could be correlated with changes in level of the corresponding enzyme activities. Recently, Devarenne et al. (2002) reported that suppression of SS enzyme activity is correlated with the corresponding mRNA level in suppression of sterol biosynthesis by fungal elicitor treatment. Further efforts are underway to test whether three triterpene biosynthetic pathway genes are activated by the T-DNA tag in activation tagging hairy root line 11-12. It is a straightforward method that we can prove linkage between the activation tag and overexpressed gene by analyzing the effects of retransformation of the recovered ginseng genomic DNA into wild type plant (van der Fits and Memelink 2000). 
Acknowledgments This work was supported by a grant to DWC from Plant Diversity Research Center of 21st Century Frontier Research Program and a grant to JRL from the Crop Functional Genomics Center of the 21st Century Frontier Research Program, and a grant to JRL from Studies on Molecular Genomics of Marine and Extreme Organisms funded by the Korean Ministry of Marine Affairs and Fisheries.

\section{References}

Ahn JC, Hwang B, Tada H, Ishimaru K, Sasaki K, Shimomura K (1996) Polyacetylenes in hairy roots of Platycodon grandiflorum. Phytochemistry 42:69-72

Azpiroz-Leehan R, Feldman K(1997) T-DNA insertion mutagenesis in Arabidopsis: going back and forth. Trend Genet 13:152-156

Bonhomme V, Laurain-Mattar D, Lacoux J, Afliniaux M, Jacquin Dubreuil A (2000) Tropane alkaloid production by hairy roots of Atropa bellodonna obtained transformation with Agrobacterium rhizogenes 15834 and Agrobacterium tumefaciens containing rolA, B, C genes only. J Biotechnol 81:151-158

Borevitz KO, Xia Y, Blount J, Dixon RA, Lam C (2000) Activation tagging identifies a conserved MYB regulator of phenylpropanoid biosynthesis. Plant Cell 12:2383-2393

Bouche N, Bouchez D (2001) Arabidopsis gene knokout: Phenotypes wanted. Curr Opinn Plant Biol 4:111-117

Bouchez D, Hofte H (1998) Functional genomics in plants. Plant Physiol 118:725-732

Bulgakov VP, Khodakovskaya MV, Labetskaya NV, Chernoded GK, Zhuravlev YN (1998) The impact of plant rolcC oncogene on ginsenoside production by ginseng hairy root cultures. Phytochemistry 49:1929-1934

Croteau R, Kutchan TM, Lewis NG (2000) Natural products (secondary metabolites), In: BB Buchanan, W Grussem, RL Jones, (eds), Biochemistry and Molecular biology of Plants, Am Soc Plant Physiol, pp 1250-1318

Cunillera N, Arro M, Delourme D, Karst F, Boronat A, Ferrer A (1996) Arabidopsis thaliana contains two differentially expressed farnesyl-diphosphate synthase genes. J Bio Chem 271:7774-7780

Devarenne TP, Ghosh A, Chappell J (2002) Regulation of squalene synthase, a key enzyme of sterol biosynthesis, in Tobacco. Plant Physiol 129:1095-1106

Dixon R (1999) Plant natural products: the molecular genetic basis of biosynthetic diversity. Curr Opin Biotechnol 10:192-197

Fiehn O, Kopka J, Dormann P, Altmann T, Trethewey RN, Willmitzer L (2000) Metabolic profiling for plant functional genomics. Nat Biotechnol 18:1157-1161

Gaffe J, Bru JP, Causse M, Vidal A, Stamitti-Bert L, Carde JP, Callusci P (2000) LEFPS1, a tomato fernesyl phrophosphate gene highly expressed during early fruit development. Plant Physiol 123: 1351-1362

Huang S, Cerny RE, Bhat DS, Brown SM (2001) Cloning of an Arabidopsis patatin-like gene STURDY, by activation T-DNA tagging. Plant Physiol 125:573-584
Kakimoto T (1996) CkiI, a histone kinase homology implicated in cytokinin signal transduction. Science 274:982-985

Mallol A, Cusido RM, Palazon J, Bonfill M, Morales C, Pinol MT (2001) Ginsenoside production in different phenotypes of Panax ginseng transformed root. Phytochemistry 57:365-371

Moyano E, Fornale S, Palazon J, Cusido RM, Bonfill M, Morales C, Pinol MT (1999) Effect of Agrobacterium rhizogenes T-DNA on alkaloid production in Solanaceae plants. Phytochemistry 52:1287-1292

Nilsson O, Olsson O (1997) Getting to the root: The role of the Agrobacterium rhizogenes rol genes in the formation of hairy roots. Physiol Plant 100:463-473

Pichersky E, Gang D (2000) Genetics and biochemistry of secondary metabolites in plants: an evolutionary perspective. Trend Plant Sci 5:439-445

Raamsdonk LM, Teusink B, Broadhurst D, Zhang N, Hayes A, Walsh MC, Berden JA, Brindle KM, Kell DB, Rowland JJ, Westerhoff HV, van Dam K, Oliver SG (2001) A functional genomics strategy that uses metabolome data to reveal the phenotype of silent mutations. Nat Biotechnol 19:45-50

Roessner U, Luedemann A, Brust D, Fiehn O, Linke T, Willmitzer L, Fernie AR (2001) Metabolic profiling allows comprehensive phenotyping of genetically or environmentally modified plant systems. Plant Cell 13:11-29

Spena A, Schmulling T, Koncz C, Schell JS (1987) Independent and synergistic activity of rol A, B and C loci in stimulating abnormal growth in plants. EMBO J 6:3891-3899

Sumner LW, Duran AL, Huhman DV, Smith JT (2002) Metabolomics: a developing and integral component in functional genomic studies of Medicago truncatula. In JT Romeo, RA Dixon, (eds), Phytochemistry in the Genomics and Post-Genomics Eras. Elsevier, Oxford, pp 31-61

Trethewey RN (2001) Gene discovery via metabolic profiling. Curr Opin Biotehcnol 12:135-138

van der Fits L, Hilliou F, Memelink J (2001) T-DNA activation tagging as a tools to isolate regulators of a metabolic pathway from a genetically non-tractable plant species. Trans Res 10: 513-521

van der Fits L, Memelink J (2000) ORCA3, a jasmonate-responsive transcriptional regulator of plant primary and secondary metabolism. Science 289: 295-297

Walden R, Fretze K, Hayashi H, Miklashevichs E, Harling H, Shell J (1994) Activation tagging: a means of isolating genes implicated as playing a role in plant growth and development. Plant Mol Biol 26:1521-1528

Weigel D, Ahn JH, Blázquez MiA, Borevitz JO, Christensen SK, Fankhauser C, Ferrándiz C, Kardailsky I, Malancharuvil EJ, Neff MM, Nguyen JT, Sato S, Wang ZY, Xia Y, Dixon RA, Harrison MJ, Lamb CJ, Yanofsky MF, and Chory J (2000) Activation tagging in Arabidopsis. Plant Physiol 122:1003-1014

Wentzinger LF, Bach TJ, Hartmann MA (2002) Inhibition of squalene synthase and squalene epoxidase in tobacco cells triggers an up-regulation of 3-hydroxy-3-methylgultaryl coenzyme A reductase. Plant Physiol 130:334-346 